\title{
CONSTRUÇÃO E VALIDAÇÃO DA ESCALA DE COMPORTAMENTOS PARA A SUSTENTABILIDADE
}

\author{
http://dx.doi.org/10.21527/2237-6453.2021.55.11606
}

Recebido em: 13/10/2020

Aceito em: 14/4/2021

\author{
Valéria Garlet ${ }^{1}$, Thiago Antônio Beuron ${ }^{2}$, Lucas Veiga Ávila, ${ }^{1}$ \\ Laércio André Gassen Balsan, ${ }^{1}$ Lúcia Rejane da Rosa Gama Madruga ${ }^{1}$
}

\begin{abstract}
RESUMO
Este estudo tem por objetivo a construção e validação de uma Escala de Comportamentos para a Sustentabilidade (Ecos). A pesquisa adotou as seguintes etapas: desenvolvimento, análise de conteúdo, análise da confiabilidade e validade da escala para identificar os fatores determinantes dos Comportamentos para a Sustentabilidade. $\mathrm{O}$ instrumento passou por procedimentos teóricos, experimentais e analíticos durante sua validação. Participaram da pesquisa 509 indivíduos. Os resultados sustentaram a existência de três fatores: consumo consciente, preocupação com o meio ambiente e desperdício. O questionário quantitativo mostrou bons índices e evidências de validade de conteúdo e de confiabilidade, e sua utilização permitiu uma análise fidedigna dos comportamentos em prol da sustentabilidade. Tal fato permitirá um melhor entendimento de como as características individuais podem impactar de forma benéfica ou ameaçadora as futuras gerações. Conhecer esses comportamentos é fundamental para a introdução de práticas educacionais e políticas públicas e gerenciais que visem à garantia do respeito aos recursos naturais e à vida no Planeta.
\end{abstract}

Palavras-chave: Sustentabilidade. Comportamentos. Escala.

CONSTRUCTION AND VALIDATION OF THE SCALE OF BEHAVIORAL SUSTAINABILITY

\section{ABSTRACT}

This study allowed the construction and validation of the Behavior Scale for Sustainability. The research adopted the stages: development, content analysis, reliability analysis and scale validity to identify the determinants of Behaviors for Sustainability. The instrument underwent theoretical, experimental and analytical procedures. 509 individuals participated in the research. The results supported the existence of three factors: conscious consumption, concern for the environment and waste. The quantitative questionnaire showed good indexes and evidence of content validity and reliability, and its use allowed a reliable analysis of behaviors in favor of sustainability. This will allow a better understanding of how individual characteristics can have a beneficial or threatening impact on future generations.

Keywords: Sustainability. Behaviors. Scale.

\footnotetext{
${ }^{1}$ Autora correspondente. Universidade Federal de Santa Maria (UFSM). Av. Roraima, № 1000 - Cidade Universitária - Bairro Camobi. Santa Maria/RS, Brasil. CEP 97105-900. http://lattes.cnpq.br/6442130419963857. https://orcid.org/0000-0001-9606-6001. valeriagarlet@yahoo.com.br

2 Universidade Federal do Pampa (Unipampa).
} 
CONSTRUÇÃO E VALIDAÇÃO DA ESCALA DE COMPORTAMENTOS PARA A SUSTENTABILIDADE Valéria Garlet - Thiago Antônio Beuron - Lucas Veiga Ávila - Laércio André Gassen Balsan - Lúcia Rejane da Rosa Gama Madruga

\section{INTRODUÇÃO}

A sustentabilidade, enquanto ampliação do conceito de desenvolvimento sustentável, trata da preservação da vida no Planeta. Para que essa preservação realmente ocorra é necessário que os seres humanos, como agentes de transformação (positiva e negativa), incorporem hábitos de conservação do meio ambiente. Essa incorporação não é um objetivo simples; requer conscientização, esforço diário para manter práticas sustentáveis e mudanças nos comportamentos individuais e coletivos: "é inadiável e imprescindível que comportamentos e atitudes voltadas a um desenvolvimento sustentável local sejam eleitas como primordiais nas políticas públicas mundiais. Isto é questão de sobrevivência para a humanidade" (SALDANHA, 2019, p. 28). Conforme evidencia Costa (2017, p. 92), "ampliar a ideia de Sustentabilidade é ampliar a ideia de felicidade e de sociedade, contrapondo-se a uma visão antropocêntrica e construindo um conceito mais holístico assegurando a todos uma qualidade de vida melhor".

O consenso da Unesco (2014), durante a década para o Desenvolvimento Sustentável (DS) (2005-2014), tinha sua ênfase na educação e no aprendizado. Com base em todas as discussões e protocolos anteriores, acordados pelos Chefes de Estado e de Governo e representantes seniores reunidos na sede das Nações Unidas, em 2015 foram pactuados novos Objetivos de Desenvolvimento Sustentável (ODS) das Nações Unidas, apontando para a necessidade de uma agenda para os próximos anos (Agenda 2030). A discussão sobre tal agenda tem sido marcada por uma série de eventos, declarações e resoluções, que têm o objetivo de entender e proporcionar a inserção da sustentabilidade por intermédio de variáveis comportamentais que precisam ser inseridas no dia a dia. Esse debate sobre o desenvolvimento sustentável não é novo. Ainda assim, essa área sofre com a superabundância de textos e com a não efetivação dos acordos em sua totalidade. Com base nessa problemática, o presente estudo buscou ir além do debate teórico a fim de visualizar a sustentabilidade de maneira realizável, por meio de uma medida para as ações práticas (LEAL FILHO, 2011), mais especificamente de comportamentos voltados para a sustentabilidade.

Conforme destacam Prado, Wander e Costa Filho (2019, p. 958), "a sustentabilidade ambiental ganhou destaque frente à urgência existente na mudança do comportamento social em busca de melhores condições de vida para a população. Diversos efeitos, resultantes da conduta humana inconsequente, são fortemente percebidos atualmente". Diversos estudos têm explorado os comportamentos humanos relacionados ao contexto do desenvolvimento sustentável e do meio ambiente. Entre os conceitos que se aproximam dos buscados neste trabalho podem ser destacados: comportamento ecológico (AXELROD; LEHMAN, 1993; KAISER; WÖLFING; FUHRER, 1999; PATO, 2004); comportamento pró-ambiental (KARP, 1996; CORRAL-VERDUGO; PINHEIRO, 1999; CORRAL-VERDUGO, 2000; BONNES; BONAIUTO, 2002); comportamento ambientalmente significante (STERN, 2000); e comportamento ecológico responsável (CALVO-SALGUERO; AGUILAR-LUZÓN; BERRIO-MARTOS, 2008).

$\mathrm{Na}$ tentativa de compreender os comportamentos humanos ante ao meio ambiente, e considerando que "os comportamentos pró-ambientais ajudam a minimizar o impacto negativo do homem ao meio ambiente" (NASCIMENTO, 2019a, p. 27), estudos têm buscado estabelecer relações entre valores e variáveis de perfil como elementos que predizem uma conduta pró-ambiental. No campo da psicologia ambiental, as pesquisas ganharam destaque ao investigar as influências mútuas entre o comportamento e o meio ambiente, além de buscarem compreender 
CONSTRUÇÃO E VALIDAÇÃO DA ESCALA DE COMPORTAMENTOS PARA A SUSTENTABILIDADE Valéria Garlet - Thiago Antônio Beuron - Lucas Veiga Ávila - Laércio André Gassen Balsan - Lúcia Rejane da Rosa Gama Madruga

as condições que originam os comportamentos (DARLEY; GILBERT, 1985). A partir do avanço das pesquisas com medidas mais gerais, surgiram medidas específicas voltadas para diferentes realidades. Algumas focalizadas nos comportamentos e atitudes, outras nas crenças dos indivíduos. Visando a contribuir para a compreensão do comportamento dos indivíduos, este artigo tem por objetivo a construção e validação de uma Escala de Comportamentos para a Sustentabilidade (Ecos).

Validar uma escala de comportamentos que podem ser usados para avaliar as atitudes dos indivíduos diante da sustentabilidade em diferentes organizações e contextos, é essencial para verificar quais indivíduos tendem a se sentir mais estimulados, participativos e comprometidos com iniciativas sustentáveis. Tal conhecimento permitirá práticas gerencias e políticas públicas e educacionais transformadoras da sociedade como um todo, visando a um futuro melhor para as gerações futuras. A seguir apresenta-se os aspectos teóricos, método do estudo, resultados e considerações finais.

\section{ESCALAS DE COMPORTAMENTOS PARA A SUSTENTABILIDADE}

É inevitável pensar em sustentabilidade sem a relacionar com práticas, ações e hábitos que reflitam a preocupação com o meio ambiente e que, por conseguinte, representam os comportamentos para a sustentabilidade. $O$ instituto Akatu (2013) destaca os comportamentos para o consumo consciente em favor do meio ambiente: economia: não desperdício de recursos, resultando em benefícios diretos e imediatos ao consumidor; planejamento: uso racional dos recursos, gerando melhor aproveitamento e maior retorno; reciclagem: descarte correto, reuso e reaproveitamento de materiais, gerando retorno a longo prazo para a sociedade; compras sustentáveis: práticas que refletem a conscientização dos consumidores e sua preocupação com as causas sustentáveis.

É possível inferir que "a mudança no comportamento humano implica minimizar as posições individualizadas colocando, acima das mesmas, um comportamento coletivo que será necessário para enfrentar as soluções compartilhadas a serem encaminhadas" (MADRUGA, 2009, p. 17). Dessa forma, cada indivíduo não pode pensar apenas nas suas práticas, mas também refletir sobre o que a sociedade em geral pode fazer em prol da sustentabilidade, como desenvolver projetos de conscientização sobre a separação e reciclagem do lixo na comunidade ou projetos voltados para outras áreas da sustentabilidade, atuação nas escolas da região, parcerias com o poder público local para melhorar as condições físicas naturais da cidade, parcerias com empresas privadas para desenvolver programas sustentáveis nos mais diversos contextos e, acima de tudo, cada pessoa deve ter a noção de que suas ações, por menores que sejam, e sejam elas positivas ou negativas, acabam gerando automaticamente uma série de impactos e consequências. A atenção que é dada às necessidades e desejos individuais, juntamente com o enaltecimento do hedonismo, evidenciam um comportamento voltado para o individual. 0 esforço na busca pelo consumo consciente, no entanto, provoca o consumidor, que pensa no individual, a pensar no bem-estar coletivo e a consumir de forma sustentável e cidadã (PINTO; BATINGA, 2016).

Ao retomar e analisar o conceito de desenvolvimento sustentável, Feil e Schreiber (2017) destacam que não é apresentada uma maneira específica para preservar o meio ambiente, mas o que fica claro é a necessidade de mudanças nos comportamentos das pessoas em prol dos 
CONSTRUÇÃO E VALIDAÇÃO DA ESCALA DE COMPORTAMENTOS PARA A SUSTENTABILIDADE Valéria Garlet - Thiago Antônio Beuron - Lucas Veiga Ávila - Laércio André Gassen Balsan - Lúcia Rejane da Rosa Gama Madruga

objetivos propostos no conceito inicial sobre o desenvolvimento sustentável. Para os autores, o conceito de desenvolvimento sustentável está atrelado a estratégias de longo prazo que englobem as perspectivas social, ambiental e econômica e que visem à qualidade de vida da sociedade. Estratégia é formulada "com base nos resultados das avaliações da sustentabilidade, e tem como foco os aspectos negativos, recuperando ou normalizando até o ponto em que o processo evolutivo do sistema ocorra normalmente" (FEIL; SCHREIBER, 2017, p. 676).

Lehman e Geller (2004) apresentam a crise ambiental nas seguintes ameaças: poluição do ar, mudanças climáticas, poluição da água, lixo sólido, erosão do solo e contaminação, perda de espaço verde e diminuição da diversidade das espécies, aquecimento global, superpopulação, aterros sanitários, destruição da camada de ozônio. Esses problemas "são principalmente, se não exclusivamente, causados pelo comportamento humano" (LEHMAN; GELLER, 2004, p. 17). Os autores propõem, em seu trabalho, estratégias de intervenção para mudança de comportamento. Como estratégias antecedentes tem-se:

- Informação e educação: informações isoladas podem não ser suficientes para a mudança de comportamento. Uma abordagem intervencionista pode auxiliar nestas questões.

- Estratégias de solicitação: mensagens que informam comportamentos desejados.

- Modelagem: demonstração de um comportamento pró-ambiental desejado para um determinado público.

- Comprometimento: o participante compromete-se em realizar determinado comportamento.

- Desenho ambiental: inserção de objetos ou dispositivos que oportunizam comportamentos pró-ambientais.

Como estratégias resultantes, Lehman e Geller (2004) sugerem: recompensas e feedback: "ao expandir o escopo das metas comportamentais, abordar o problema da manutenção a longo prazo e disseminar amplamente o que aprendemos, uma abordagem analítica do comportamento revitalizada pode atingir seu potencial de ajudar a preservar o meio ambiente (LEHMAN; GELLER, 2004, p. 27).

O estudo de Vicente-Molina et al. (2013) analisou a influência do conhecimento ambiental sobre o comportamento pró-ambiental entre estudantes universitários de países com diferentes níveis de desenvolvimento econômico (EUA, Espanha, México e Brasil). O objetivo do trabalho foi evidenciar as variáveis sociodemográficas e psicológicas que, aparentemente, influenciam o comportamento pró-ambiental. Como resultados do estudo tem-se que os fatores externos (cultura, estruturas e serviços ambientais em cada país) desempenham papel relevante no comportamento ambiental; também a motivação e eficácia percebida são importantes na explicação do comportamento em prol do meio ambiente. A educação formal e o conhecimento das questões ambientais influenciam o comportamento pró-ambiental de forma complexa.

Pato e Tamayo (2006) estudaram o comportamento ecológico com 234 estudantes de Ensino Médio e universitário e propuseram uma escala que retrata a multidimensionalidade do comportamento ecológico baseada em quatro fatores principais: ativismo-consumo, economia de água e de energia, limpeza urbana e reciclagem. Os autores salientam que é importante que se construa instrumentos confiáveis e adequados para medir o comportamento ecológico na realidade brasileira. 
CONSTRUÇÃO E VALIDAÇÃO DA ESCALA DE COMPORTAMENTOS PARA A SUSTENTABILIDADE Valéria Garlet - Thiago Antônio Beuron - Lucas Veiga Ávila - Laércio André Gassen Balsan - Lúcia Rejane da Rosa Gama Madruga

O comportamento pró-ambiental tem relação direta com o cumprimento de ações específicas e eficazes que satisfazem as necessidades sociais e individuais e que têm como resultado a preservação do meio ambiente (CORRAL-VERDUGO, 2001). Além disso, o comportamento pró-ambiental surge da necessidade de preservação dos recursos naturais; quando se percebe que estes são finitos, e se começa a propor previsões de até quando eles vão durar, inicia-se uma discussão sobre como está se dando a utilização destes recursos: questiona-se as práticas de produção, o consumo excessivo e as escolhas que estão sendo decididas em relação ao aproveitamento dos recursos naturais (AFONSO, 2013, p. 27).

Ribeiro e Veiga (2011) apresentam uma escala para identificar comportamentos do consumidor em prol de um consumo sustentável - este, "conceitualmente concebido como a consciência ecológica na compra de produtos e serviços, o não desperdício de recursos, o empenho em reciclagem de materiais e produtos e a propensão para um estilo de vida menos consumista" (RIBEIRO; VEIGA, 2011, p. 58). A escala deste estudo abrange quatro dimensões: consciência ecológica, economia de recursos, reciclagem e frugalidade; esta escala analisa traços da personalidade e o consumo sustentável e pode propiciar meios de influenciar as pessoas para que elas adotem comportamentos positivos em relação à preservação do meio ambiente.

Rosa, Leonidio e Jesus (2015) aplicaram uma Escala de Comportamento Ecologicamente Consciente do Consumidor, com o objetivo de entender como a consciência ecológica influencia os hábitos dos consumidores e seus comportamentos de compra. A escala utilizada neste estudo está composta por três fatores:

- Reciclagem: eu procuro comprar produtos feitos de papel; eu tento comprar apenas produtos que podem ser reciclados; sempre que possível eu compro produtos feitos com material reciclados; eu evito comprar produtos com embalagens que não são biodegradáveis; eu procuro não comprar produtos que possuem grandes quantidades de embalagens recicláveis; eu sempre faço um esforço para reduzir o uso de produtos feitos de recursos naturais escassos; eu não compro produtos para minha casa que prejudiquem o meio ambiente.

- Mudança de hábito: quando eu tenho que escolher entre dois iguais, eu sempre escoIho o que é menos prejudicial às outras pessoas e ao meio ambiente; eu já convenci amigos e parentes a não comprar produtos que prejudiquem o meio ambiente; quando eu conheço os possíveis danos que um produto pode causar ao meio ambiente, eu não compro esse produto; eu não compro produtos e alimentos que podem causar a extinção de algumas espécies animais ou vegetais; eu já troquei ou deixei de usar produtos feitos por razões ecológicas; eu não compro produtos fabricados ou vendidos por empresas que prejudicam ou desrespeitam o meio ambiente.

- Saúde: eu compro produtos orgânicos porque são mais saudáveis; eu prefiro produtos e alimentos sem agrotóxicos porque eles respeitam o meio ambiente; quando eu compro produtos e alimentos as preocupações com o meio ambiente interferem na decisão de compra.

Ainda, segundo esta pesquisa, "consumidor consciente é aquele que ao satisfazer suas necessidades de consumo, consegue fazer valer seu poder de compra de maneira conscientemente, não prejudicando o ambiente e satisfazendo a necessidades como indivíduo enquanto atua como cidadão" (ROSA; LEONIDIO; JESUS, 2015, p. 11). 
CONSTRUÇÃO E VALIDAÇÃO DA ESCALA DE COMPORTAMENTOS PARA A SUSTENTABILIDADE Valéria Garlet - Thiago Antônio Beuron - Lucas Veiga Ávila - Laércio André Gassen Balsan - Lúcia Rejane da Rosa Gama Madruga

Faria et al. (2018) estudaram a influência do conhecimento sobre a sustentabilidade nas atitudes, comportamentos e consumo de 131 estudantes de administração. Como achados, os autores destacaram que o conhecimento sobre sustentabilidade influencia positivamente as atitudes dos alunos, o comportamento deles em relação à sustentabilidade e o consumo sustentável dos participantes. Estes resultados ampliam a discussão sobre o tema da sustentabilidade para que estes alunos, como cidadãos e futuros profissionais da administração, possam ser capazes de mudar para melhor a situação do planeta por meio das suas atitudes e comportamentos sustentáveis.

Benedicto, Siqueira e Milani (2018) analisaram o comportamento de 67 estudantes universitários de design de interiores e identificaram que os estudantes acreditam que o uso de materiais ecológicos influencia a relação das pessoas com o meio. Eles possuem uma visão positiva em relação a esse tipo de material sustentável, porém não se sentem prontos para utilizá-los em seus projetos. Para que as mudanças voltadas para a sustentabilidade aconteçam, segundo as autoras, é preciso educação ambiental e práticas eficazes no ensino.

Melo et al. (2018) estudaram as atitudes e comportamentos para a sustentabilidade de 269 alunos do curso de administração da Universidade Federal do Ceará e encontraram que estes consideram suas atitudes ambientalmente corretas, preocupadas com a gravidade dos problemas ambientais do planeta, e não depositam confiança nos comportamentos adotados pelas organizações, ou seja, veem o nível de compromisso das organizações baixo, especialmente das empresas de produtos embalados. Mesmo ao se considerarem com atitudes conscientes, porém, a maior parte dos estudantes não adota comportamentos pró-ambientais. Os autores retratam a necessidade de inserir a sustentabilidade nos currículos, não somente da administração, mas em todas as áreas, para que a formação dos profissionais seja baseada na sustentabilidade.

Prado, Wander e Costa Filho (2019) estudaram, por meio do método survey, o comportamento e a percepção da sustentabilidade em instituições bancárias públicas e privadas com base nas políticas já existentes nas organizações, e identificaram que os empregados de instituições privadas apresentam uma percepção positiva e uma predisposição a praticar a sustentabilidade no ambiente organizacional, com comportamentos alinhados às políticas da empresa. Mesmo, todavia, havendo conhecimento, predisposição e comportamentos favoráveis à sustentabilidade, os autores perceberam que há espaço para os empregados se envolverem em ações proativas nas organizações em que trabalham.

Nascimento (2019) estudou a relação do comportamento pró-ambiental com o bem-estar subjetivo e revelou que, ao ter atitudes sustentáveis nas compras, os consumidores têm um aumento no seu bem-estar subjetivo; o bem-estar subjetivo também é alcançado quando há, além do comportamento pró-ambiental, um compromisso com a igualdade, justiça e proteção de todos. A autora ainda evidencia que "é possível ser otimista, há uma crescente conscientização de que se enfrenta problemas ambientais globais e que mudanças significativas nas políticas, tecnologias e comportamentos serão necessárias para abordá-lo" (NASCIMENTO, 2019b, p. 93).

Sob um olhar bastante preocupante, Costa (2017, p. 89) analisa a relação entre consumo e sustentabilidade com 150 jovens, e destaca que "os jovens se enxergam como seres separados da natureza, como se não fossem parte integrante de um todo". Para estes jovens, o ambiente está relacionado a floretas e zonas rurais. Eles se veem num cenário longe da natureza e 
desejam o consumo moderno, focando no preço, na durabilidade e na qualidade dos produtos na hora da compra, não pensando em questões no âmbito da responsabilidade socioambiental. A pesquisa da autora mostrou que os jovens analisados não compram em brechós e não reaproveitam roupas ou materiais eletrônicos; têm certa antipatia por produtos reciclados e não costumam separar resíduos para reciclagem: "a forma como os jovens consomem reflete a maneira como eles enxergam a crise ambiental, ou seja, como algo dissociado da sociedade, sobre a qual não têm poder ou responsabilidade" (COSTA, 2017, p. 91). Segundo a autora, esses jovens buscam o mundo do trabalho para garantir sua sobrevivência e o acesso aos bens e serviços que desejam. Com isso, a educação torna-se uma ferramenta imprescindível para alcançar esse público e torná-lo crítico e responsável.

O presente estudo descreve a construção e a validação de uma Escala de Comportamentos para a Sustentabilidade (Ecos). Os itens do instrumento foram resgatados da literatura com base em uma ampla revisão nacional e internacional de autores que estudam ou estudaram os comportamentos para a sustentabilidade. A seguir, apresenta-se a compilação dos comportamentos encontrados na literatura e as fases da pesquisa que geram a validação da escala.

\section{MÉTODO DO ESTUDO}

Este trabalho baseia-se em uma pesquisa quantitativa, com abordagem descritiva, que foi realizado por meio do método survey. As etapas da pesquisa serão descritas a seguir, trazendo os itens que compuseram o instrumento inicial para culminar com a escala validada de comportamentos para a sustentabilidade.

O instrumento foi construído com base nos constructos teóricos acerca da sustentabilidade, itens desenvolvidos por autores amplamente citados na literatura nacional e mundial e na inclusão de variáveis adicionais propostas pelos próprios autores, conforme demonstrado no Quadro 1.

Quadro 1 - Itens considerados na construção da escala de comportamentos para a sustentabilidade

\begin{tabular}{|l|l|}
\hline Comportamentos & Autores \\
\hline Evito desperdício de energia & Pato e Tamayo (2006), Beuron (2016), Garlet (2017) \\
\hline Evito desperdício dos recursos naturais & Pato e Tamayo (2006), Beuron (2016), Garlet (2017) \\
\hline Economizo água quando possível & Pato e Tamayo (2006), Beuron (2016), Garlet (2017) \\
\hline Apago a luz quando saio de ambientes vazios & Pato e Tamayo (2006), Beuron (2016), Garlet (2017) \\
\hline $\begin{array}{l}\text { Evito ligar vários aparelhos elétricos ao mesmo } \\
\text { tempo nos horários de maior consumo de energia }\end{array}$ & Pato e Tamayo (2006), Beuron (2016), Garlet (2017) \\
\hline Evito o desperdício de alimentos & $\begin{array}{l}\text { Trivedi (2015), Thomashow (2014), Beuron (2016), } \\
\text { Garlet (2017) }\end{array}$ \\
\hline $\begin{array}{l}\text { Desligo aparelhos eletrônicos quando não estou } \\
\text { usando }\end{array}$ & Melo et al. (2017), Akatu (2013) \\
\hline $\begin{array}{l}\text { Tomo banho no menor tempo possível visando a a } \\
\text { preservar a água }\end{array}$ & Pato e Tamayo (2006) \\
\hline $\begin{array}{l}\text { Procuro comprar eletrodomésticos e equipamentos } \\
\text { com um menor consumo energia }\end{array}$ & $\begin{array}{l}\text { Roberts (1996), Straughan e Roberts (1999), Lages } \\
\text { e Vargas Neto (2002), Ribeiro e Veiga (2011) }\end{array}$ \\
\hline $\begin{array}{l}\text { Prefiro usar uma sacola/bolsa reutilizável para fazer } \\
\text { compras }\end{array}$ & Ribeiro e Veiga (2011) \\
\hline
\end{tabular}


CONSTRUÇÃO E VALIDAÇÃO DA ESCALA DE COMPORTAMENTOS PARA A SUSTENTABILIDADE Valéria Garlet - Thiago Antônio Beuron - Lucas Veiga Ávila - Laércio André Gassen Balsan - Lúcia Rejane da Rosa Gama Madruga

\begin{tabular}{|c|c|}
\hline Preocupo-me com o uso consciente da água & Proposição dos autores \\
\hline Evito jogar lixo no chão & Pato e Tamayo (2006), Beuron (2016), Garlet (2017) \\
\hline $\begin{array}{l}\text { Quando não encontro uma lixeira por perto guardo } \\
\text { o resíduo para descartar em local mais adequado }\end{array}$ & Pato e Tamayo (2006), Beuron (2016), Garlet (2017) \\
\hline Em minha casa separo o lixo para reciclagem & Melo et al. (2017), Akatu, (2013) \\
\hline Reutilizo produtos e embalagens & Akatu (2013), Pinto e Batinga (2016) \\
\hline $\begin{array}{l}\text { Prefiro produtos com embalagens que podem ser } \\
\text { recicladas }\end{array}$ & $\begin{array}{l}\text { Maloney, Ward e Braucht (1975), Roberts (1996), } \\
\text { Ottman (1998), Straughan e Roberts (1999), Thapa } \\
\text { (1999), Lages e Vargas Neto (2002), Ribeiro e Veiga } \\
\text { (2011) }\end{array}$ \\
\hline $\begin{array}{l}\text { Antes de descartar um produto/equipamento } \\
\text { procuro consertá-lo, desde que o conserto seja } \\
\text { possível e viável economicamente }\end{array}$ & Proposição dos autores \\
\hline Colaboro com a preservação da cidade onde vivo & Pato e Tamayo (2006), Beuron (2016), Garlet (2017) \\
\hline $\begin{array}{l}\text { Mobilizo as pessoas nos cuidados necessários para } \\
\text { a conservação dos espaços públicos }\end{array}$ & Pato e Tamayo (2006), Beuron (2016), Garlet (2017) \\
\hline Promovo o cuidado do meio ambiente & Pato e Tamayo (2006), Beuron (2016), Garlet (2017) \\
\hline $\begin{array}{l}\text { Procuro influenciar as pessoas para que sejam } \\
\text { cuidadosas em relação ao meio ambiente }\end{array}$ & $\begin{array}{l}\text { Thapa (1999), Gonçalves-Dias et al. (2009), Ribeiro } \\
\text { e Veiga (2011) }\end{array}$ \\
\hline $\begin{array}{l}\text { Sinto vontade de chamar a atenção de pessoas que } \\
\text { jogam lixo na rua }\end{array}$ & Pato e Tamayo (2006) \\
\hline $\begin{array}{l}\text { Colaboro para a preservação/conservação dos } \\
\text { espaços públicos }\end{array}$ & Pato e Tamayo (2006) \\
\hline $\begin{array}{l}\text { Apoio manifestações públicas em defesa do meio } \\
\text { ambiente }\end{array}$ & Pato e Tamayo (2006) \\
\hline Preocupo-me com a destruição do meio ambiente & Enoki et al. (2008), Afonso (2013) \\
\hline $\begin{array}{l}\text { Busco orientar outras pessoas a não comprarem } \\
\text { produtos que prejudicam o meio ambiente }\end{array}$ & Straughan e Roberts (1999) \\
\hline Ajudo a manter as ruas limpas. & Gonçalves-Dias et al. (2009), Afonso (2013) \\
\hline Preservo rios, mares, praias, matas ou florestas & $\begin{array}{l}\text { Instrumento do Conselho Empresarial para o } \\
\text { Desenvolvimento Sustentável - CEBDS (2010), } \\
\text { Afonso (2013) }\end{array}$ \\
\hline $\begin{array}{l}\text { Participo de discussões ou conversas sobre como } \\
\text { contribuir para uma sociedade melhor e um meio } \\
\text { ambiente mais limpo }\end{array}$ & $\begin{array}{l}\text { Instrumento do Conselho Empresarial para o } \\
\text { Desenvolvimento Sustentável - CEBDS (2010), } \\
\text { Afonso (2013) }\end{array}$ \\
\hline $\begin{array}{l}\text { Interesso-me por questões relacionadas ao meio } \\
\text { ambiente }\end{array}$ & $\begin{array}{l}\text { Maloney, Ward e Braucht (1975), Thapa (1999), } \\
\text { Ribeiro e Veiga (2011) }\end{array}$ \\
\hline $\begin{array}{l}\text { Se hoje eu fosse comprar um carro levaria em conta } \\
\text { o seu consumo }\end{array}$ & Akatu (2013) \\
\hline $\begin{array}{l}\text { Preocupo-me com a emissão de gases poluentes } \\
\text { decorrentes do uso de automóveis }\end{array}$ & Proposição original dos autores deste trabalho \\
\hline $\begin{array}{l}\text { Quando possível, eu caminho, ando de bicicleta, } \\
\text { utilizo carona ou, ainda, uso transporte público } \\
\text { para ajudar a reduzir a poluição do ar }\end{array}$ & $\begin{array}{l}\text { Mohr e Webb (2005), Magalhães (2007), Tódero } \\
\text { (2009), Afonso (2013) }\end{array}$ \\
\hline $\begin{array}{l}\text { Costumo planejar as compras de alimentos para } \\
\text { não haver desperdício }\end{array}$ & Melo et al. (2017), Akatu, (2013) \\
\hline $\begin{array}{l}\text { Em geral, ao comprar roupas, costumo comprar } \\
\text { somente o necessário }\end{array}$ & Melo et al. (2017), Akatu, (2013) \\
\hline $\begin{array}{l}\text { Costumo pedir nota/cupom fiscal quando vou às } \\
\text { compras, mesmo que o fornecedor não a ofereça } \\
\text { espontaneamente }\end{array}$ & Melo et al. (2017), Akatu, (2013) \\
\hline
\end{tabular}


CONSTRUÇÃO E VALIDAÇÃO DA ESCALA DE COMPORTAMENTOS PARA A SUSTENTABILIDADE Valéria Garlet - Thiago Antônio Beuron - Lucas Veiga Ávila - Laércio André Gassen Balsan - Lúcia Rejane da Rosa Gama Madruga

\begin{tabular}{|c|c|}
\hline $\begin{array}{l}\text { Quando possível, utilizo os dois lados das folhas de } \\
\text { papel }\end{array}$ & Melo et al. (2017), Akatu, (2013) \\
\hline $\begin{array}{l}\text { Procuro passar ao maior número possível de } \\
\text { pessoas as informações que aprendo sobre } \\
\text { empresas e produtos }\end{array}$ & Melo et al. (2017), Akatu, (2013) \\
\hline $\begin{array}{l}\text { Comprei muitos produtos orgânicos nos últimos } \\
\text { seis meses }\end{array}$ & Melo et al. (2017), Akatu, (2013) \\
\hline $\begin{array}{l}\text { Comprei produtos feitos com material reciclado } \\
\text { nos últimos seis meses }\end{array}$ & Melo et al. (2017), Akatu, (2013) \\
\hline Procuro consumir apenas o necessário & Melo et al. (2017), Akatu, (2013) \\
\hline Não compro produtos piratas ou contrabandeados & Akatu (2008), Pinto e Batinga (2016) \\
\hline $\begin{array}{l}\text { Evito comer alimentos que contenham produtos } \\
\text { químicos (conservantes ou agrotóxicos) }\end{array}$ & Pato e Tamayo (2006), Beuron (2016), Garlet (2017) \\
\hline Prefiro comprar produtos ambientalmente corretos & Akatu (2013) \\
\hline $\begin{array}{l}\text { Procuro comprar de empresas com boa imagem } \\
\text { socioambiental }\end{array}$ & Akatu (2013) \\
\hline $\begin{array}{l}\text { Acredito que a comunicação realizada pelas } \\
\text { empresas sobre responsabilidade social e ambiental } \\
\text { deve ser confiável }\end{array}$ & Akatu (2013) \\
\hline $\begin{array}{l}\text { Espero que as empresas façam mais do que está } \\
\text { estabelecido em leis, buscando trazer benefícios } \\
\text { para a sociedade }\end{array}$ & Akatu (2013) \\
\hline $\begin{array}{l}\text { Mudo meu comportamento de compra quando } \\
\text { descubro práticas negativas da empresa } \\
\text { (propaganda enganosa, não tratar bem os } \\
\text { funcionários, tem impacto social e ambiental } \\
\text { negativo...) }\end{array}$ & Akatu (2013) \\
\hline $\begin{array}{l}\text { Estou disposto a pagar mais por produtos } \\
\text { produzidos por empresas preocupadas com a } \\
\text { preservação ambiental }\end{array}$ & Proposição dos autores \\
\hline
\end{tabular}

Fonte: Elaborado pelos autores.

A primeira versão do instrumento foi composta por 59 itens distribuídos para avaliação dos seguintes fatores teóricos: Desperdício (14 itens), resíduos (15 itens), preocupação com o meio ambiente (15 itens) e consumo consciente (15 itens).

Seguindo-se os ensinamentos de Pasquali (1999), esta primeira concepção de instrumento foi submetida a uma análise semântica por meio de três sessões de brainstorming, que, segundo o autor, é a maneira que tem se mostrado mais eficaz na avaliação da compreensão dos itens. As sessões foram compostas por quatro indivíduos cada. Durante todas as sessões foram apresentados pelo pesquisador item por item, pedindo que ele fosse reproduzido pelos membros do grupo. Se a reprodução do grupo não deixasse dúvida o item era mantido; se surgissem divergências ou se o item fosse entendido de maneira diferente do que o pesquisador pretendia, o mesmo era adaptado ou excluído.

Durante essa etapa foi identificada a necessidade de apresentar o conceito de sustentabilidade junto ao instrumento, pois muitas pessoas, até mesmo tendo uma base sobre o tema, acabam pensando que sustentabilidade apenas refere-se à questão ambiental, e isso prejudicava o entendimento dos sujeitos, principalmente quando o assunto é o comportamento. 
CONSTRUÇÃO E VALIDAÇÃO DA ESCALA DE COMPORTAMENTOS PARA A SUSTENTABILIDADE Valéria Garlet - Thiago Antônio Beuron - Lucas Veiga Ávila - Laércio André Gassen Balsan - Lúcia Rejane da Rosa Gama Madruga

Em seguida, todos os itens foram novamente revisados pelos pesquisadores para verificar se ainda mantinham sua validade aparente. Para verificar a validade de conteúdo, o instrumento foi submetido à avaliação por oito especialistas com conhecimento e experiência na área, que atenderam a um dos seguintes critérios: ser pesquisador de renome nacional sobre sustentabilidade ou ter conhecimento em pesquisas com referenciais teóricos sobre comportamento. Não há um número mínimo de especialistas na área requerido para estudos de validação de conteúdo, no entanto a utilização de oito especialistas foi fundamental para analisar as questões referentes à sustentabilidade e comportamento.

Os especialistas na área avaliaram o instrumento quanto ao título, itens, instruções, formato de resposta, procedimento de escores de resposta e forma de registro. A avaliação foi realizada considerando-se a pertinência dos itens, clareza e abrangência dos mesmos. Os especialistas foram ainda informados quanto à base conceitual e população-alvo. Foram mantidos inalterados os itens que apresentaram $100 \%$ de concordância quanto à sua pertinência e clareza. Os itens nos quais houve, pelo menos, uma discordância, foram discutidos com os demais especialistas até chegar-se a um consenso de sua avaliação e reestruturação.

Após a etapa de análise pelos especialistas na área, continuou-se o processo de validação do instrumento, com um pré-teste aplicado a mais 15 sujeitos. Depois dessa etapa verificou-se que não havia mais necessidade de mudanças no instrumento. Completada essa etapa, o instrumento ficou constituído por 49 itens restantes. Para a medição dos itens foi utilizada uma escala do tipo Likert de 5 pontos, em que a atribuição do número 1 representa "Discordo Totalmente" e o número 5 "Concordo Totalmente".

Ao final do instrumento acrescentaram-se perguntas solicitando aos participantes alguns dados demográficos: gênero, idade, estado civil, grau de instrução, religião e renda familiar mensal.

O instrumento foi aplicado em uma Universidade Federal que possui, aproximadamente, 12.935 alunos de Graduação, 1.546 alunos de Pós-Graduação, 930 docentes e 913 técnicos-administrativos. A instituição conta com mais de 70 cursos de Graduação oferecidos nas modalidades presencial e a distância. As unidades possuem características urbanas, suburbanas e rurais e buscam desenvolver pesquisas nas diversas áreas do conhecimento e promover a extensão universitária, caracterizando sua inserção regional mediante atuação multicampi (com 10 unidades e reitoria) na mesorregião Metade Sul do Estado do Rio Grande do Sul.

Os questionários foram disponibilizados pela internet, via e-mail, para toda a comunidade acadêmica, conforme a orientação da administração da universidade. Participaram da pesquisa 509 indivíduos. Juntamente com o questionário os participantes receberam o Termo de Consentimento Livre e Esclarecido (TCLE), com a descrição e explicação dos objetivos deste estudo.

$\mathrm{Na}$ análise dos dados realizaram-se observações estatísticas exploratórias para identificar dados omissos, outliers e adequação da amostra. Em seguida, realizou-se análises fatoriais exploratórias (AFE) e análise fatorial confirmatória (AFC). Na AFE utilizou-se o método Principal Axis Factoring (PAF), com rotação oblíqua (direct oblimin). Para a AFC, com o objetivo de testar hipóteses específicas sobre a estrutura latente do modelo, empregou-se os indicadores "goodness", os quais permitem avaliar a qualidade de ajuste do modelo (HAIR et al., 2009), como o X2 (qui-quadrado), que testa a probabilidade de o modelo teórico se ajustar aos dados. O Goodness-of-Fit index (GFI) e o Adjusted Goodness-of-Fit index (AGFi) indicam a proporção de variân- 
cia-covariância nos dados explicada pelo modelo. Outro indicador da qualidade utilizado foi o Root-Mean-square Error of Approximation (RMSEA), com seu intervalo de confiança de $90 \%$, e o Comparative Fit index (CFI), que compara o modelo estimado e o modelo nulo, considerando valores mais próximos de 1 como indicadores de ajustamento satisfatório (HAIR et al., 2009). A seção que segue apresenta os resultados encontrados na pesquisa.

\section{RESULTADOS E ANÁLISES}

Participaram do estudo 509 sujeitos da comunidade acadêmica de uma universidade que atua em 10 cidades diferentes do Estado do Rio Grande do Sul, Brasil (Alegrete, Bagé, Dom Pedrito, São Borja, São Gabriel, Itaqui, Jaguarão, Santana do Livramento, Caçapava do Sul e Uruguaiana). Desses sujeitos, $61,3 \%$ eram do sexo feminino e $38,7 \%$ do sexo masculino; em relação ao estado civil $46,6 \%$ eram casados e $44,6 \%$ solteiros. Referente à escolaridade $28,5 \%$ têm Ensino Superior completo e $25 \%$ Ensino Médio completo. Quarenta e cinco por cento (45\%) dos respondentes afirmam ser pertencentes à Classe $\mathrm{C}$ (de $\mathrm{R} \$ 2.005,00$ a $\mathrm{R} \$ 8.640,00$ ). A maior parte dos respondentes $(53,8 \%)$ é discente da instituição, seguido de $26,9 \%$ dos técnicos-administrativos em educação e $19,3 \%$ de docentes.

O teste de Esferacidade de Bartlett foi significativo, e o KMO foi igual a 0,904. Com base em tais resultados, realizou-se a AFE, cujos principais resultados podem ser encontrados na Tabela 1.

Tabela 1 - AFE da Escala de Comportamentos para a Sustentabilidade

\begin{tabular}{|c|c|c|c|c|}
\hline & \multicolumn{4}{|c|}{ Componente } \\
\hline & F1 & $\mathrm{F} 2$ & $\mathrm{~F} 3$ & $h^{2}$ \\
\hline 1. Prefiro comprar produtos ambientalmente corretos & 0,88 & & & 0,76 \\
\hline $\begin{array}{l}\text { 2. Procuro comprar de empresas com boa imagem } \\
\text { socioambiental }\end{array}$ & 0,87 & & & 0,69 \\
\hline $\begin{array}{l}\text { 3. Estou disposto a pagar mais por produtos produzidos por } \\
\text { empresas preocupadas com a preservação ambiental }\end{array}$ & 0,81 & & & 0,65 \\
\hline 4. Comprei muitos produtos orgânicos nos últimos seis meses & 0,75 & & & 0,55 \\
\hline $\begin{array}{l}\text { 5. Mobilizo as pessoas nos cuidados necessários para a } \\
\text { conservação dos espaços públicos }\end{array}$ & & 0,94 & & 0,79 \\
\hline 6. Promovo o cuidado do meio ambiente & & 0,89 & & 0,81 \\
\hline $\begin{array}{l}\text { 7. Procuro influenciar as pessoas para que sejam cuidadosas } \\
\text { em relação ao meio ambiente }\end{array}$ & & 0,79 & & 0,78 \\
\hline 8. Economizo água quando possível & & & 0,87 & \\
\hline 9. Apago a luz quando saio de ambientes vazios & & & 0,86 & \\
\hline 10. Evito desperdício dos recursos naturais & & & 0,80 & \\
\hline Autovalores & 5,36 & 1,32 & 1,10 & \\
\hline Variância explicada (\%) & 48,73 & 12,0 & 9,99 & \\
\hline Alfa de Cronbach & 0,85 & 0,86 & 0,80 & \\
\hline Número de itens & 4 & 3 & 3 & \\
\hline \multicolumn{5}{|l|}{$\begin{array}{l}\text { F1 }=\text { Consumo consciente } \\
\text { F2 = Preocupação com o meio ambiente } \\
\text { F3 = Desperdício }\end{array}$} \\
\hline \multicolumn{5}{|c|}{ Fonte: Elaborada pelos autores com base nos resultados da pesquisa. } \\
\hline
\end{tabular}


Da escala pré-testada, composta por 49 itens, divididos teoricamente em 5 fatores, restaram 10 itens divididos em 3 fatores. Como observa-se na Tabela 1, os fatores que emergiram após a AFE apresentaram valor próprio de 5,36 para o Fator 1 (Consumo consciente), 1,32 para o Fator 2 (Preocupação com o meio ambiente) e 1,10 para o Fator 3 (Desperdício), explicando, em conjunto, um total de $70,80 \%$ da variância total.

Consumo consciente (4 itens) - agrupou os itens relacionados ao comportamento de consumo de produtos ou empresas que tenham um impacto menor (exemplos: "Prefiro comprar produtos ambientalmente corretos; procuro comprar de empresas com boa imagem socioambiental).

Preocupação com o meio ambiente ( 3 itens) - juntou itens relacionados às questões do meio ambiente (exemplos: "Mobilizo as pessoas nos cuidados necessários para a conservação dos espaços públicos; promovo o cuidado do meio ambiente").

Desperdício ( 3 itens) - agrupou os itens referentes ao desperdício e economia de recursos (exemplos: "Economizo água quando possível; evito desperdício dos recursos naturais").

Considerando os resultados da AFE, realizou-se uma AFC visando a testar a adequação da escala. Assim, verificou-se que os indicadores de qualidade de ajuste foram: $\mathrm{X}^{2} / \mathrm{gl}=2,069, \mathrm{GFI}=$ $0,97, \mathrm{CFI}=0,98, \mathrm{TLI}=0,98, \mathrm{NFI}=0,97, \mathrm{RMSA}=0,04, \mathrm{RMSEA}=0,03-0,06$, confiabilidade composta 0,94 e variância extraída igual a 0,65. Esses demonstraram-se adequados e os resultados da estrutura do modelo estão sumarizados na Figura 1.

Figura 1 - Estrutura do modelo da Escala de Comportamentos para a Sustentabilidade

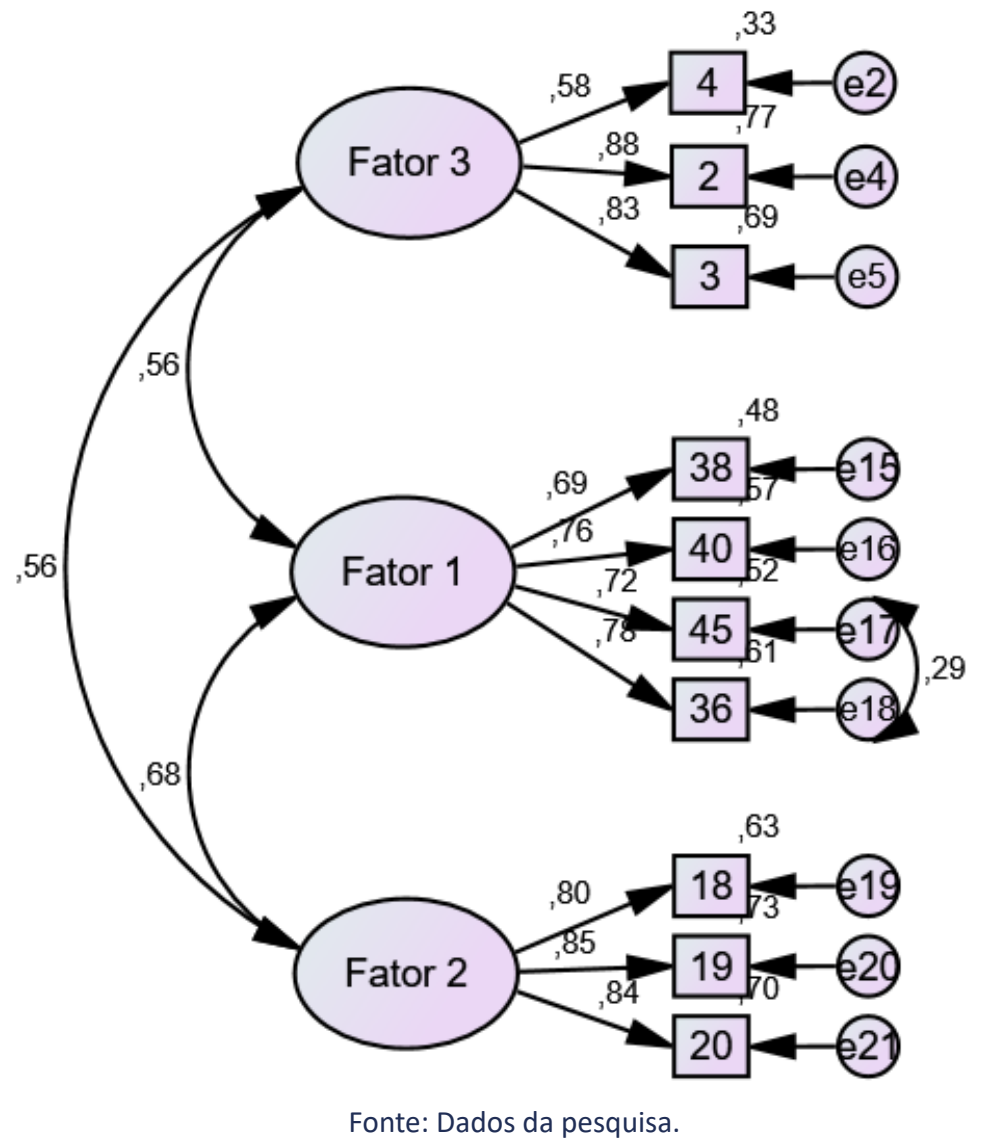


CONSTRUÇÃO E VALIDAÇÃO DA ESCALA DE COMPORTAMENTOS PARA A SUSTENTABILIDADE Valéria Garlet - Thiago Antônio Beuron - Lucas Veiga Ávila - Laércio André Gassen Balsan - Lúcia Rejane da Rosa Gama Madruga

O modelo inicial sugeriu índices de melhorias e assim foi reespecificado com a inclusão da correlação entre os erros e17 (Procuro comprar de empresas com boa imagem socioambiental) e e18 (Prefiro comprar produtos ambientalmente corretos).

Quanto à $\mathrm{AFC}$, os resultados apoiaram a $\mathrm{AFE}$, no entanto não confirmaram a estrutura composta por cinco fatores como imaginado pelos autores desses estudos e pelos especialistas na área convidados. A solução pensada era que o construto Comportamentos para Sustentabilidade seria composto por cinco fatores: Desperdício, Resíduos, Preocupação com o meio ambiente e Consumo consciente. Desses, apenas três compuseram o modelo: Fator 1 (Consumo consciente), Fator 2 (Preocupação com o meio ambiente) e Fator 3 (Desperdício).

Os índices de ajuste observados demonstraram-se apropriados (BYRNE, 2001; TABACHNICK; FIDELL, 2001; HAIR et al., 2009), e os itens apresentaram carga fatorial acima de 0,75 , com comunalidades superiores a 0,75 . Somado a isso, obteve-se níveis adequados de consistência interna dos fatores: Consumo consciente $(\alpha=0,85)$, Preocupação com o meio ambiente $(\alpha=$ $0,86)$ e Desperdício $(\alpha=0,80)$.

\section{CONSIDERAÇÕES FINAIS}

Os resultados obtidos indicam que a Ecos apresenta-se como uma ferramenta válida, compreensível e com conceitos que mostram evidência de consistência interna. Sua utilização pode subsidiar novas pesquisas, utilizando as medidas de comportamentos para a sustentabilidade e suas relações com outros constructos. Os resultados apontam para a existência de três fatores de Comportamento para a Sustentabilidade, que explicaram $70,803 \%$ da variância total. Apesar de o primeiro fator apresentar variância maior $(48,73 \%)$, os demais fatores apresentaram variância acima de $9 \%$, sendo considerados relevantes para a explicação dos Comportamentos para a Sustentabilidade.

Os três fatores que compõem o construto apresentaram índices Alfa de Cronbach acima de 0,80 , o que indica confiabilidade da medida. Os fatores expuseram cargas fatoriais satisfatórias, sendo bem representados pelos itens, os quais mostraram cargas superiores a 0,75 . Apesar disso, pesquisas futuras deverão replicar a medida aqui proposta em novos ambientes e culturas, bem como verificar a relação entre os comportamentos para a sustentabilidade e variáveis ambientais, sociais e demográficas.

Somado a isso, a escala aqui proposta poderá contribuir muito para um melhor entendimento de como características atitudinais podem ser benéficas ou ameaçadoras às mudanças climáticas, poluição, erosão do solo, desmatamento, diminuição da diversidade das espécies, aquecimento global, bem como para a vida futura da espécie humana.

Para pesquisas futuras, sugere-se a realização de estudos de casos nas Unidades Administrativas, visando a uma maior mensuração e coleta de informações. Salienta-se, também, que os avanços em prol da Agenda 2030 no atendimento dos ODSs, poderão permitir melhores indicadores de Comportamentos em organizações/instituições.

\section{AGRADECIMENTOS}

Ao Conselho Nacional de Desenvolvimento Científico e Tecnológico (CNPq) e à Fundação de Amparo à Pesquisa do Estado do RS (Fapergs). 


\section{REFERÊNCIAS}

AFONSO, M. H. F. Mensuração da predisposição ao comportamento sustentável por meio da teoria da resposta ao item. 2013. Dissertação (Mestrado) - Universidade Federal de Santa Catarina, Centro Tecnológico, Programa de Pós-Graduação em Engenharia de Produção, Florianópolis, 2013.

AKATU. Pesquisa AKATU 2012: rumo à sociedade do bem-estar. Percepção da responsabilidade social empresarial pelo consumidor brasileiro. 1. ed. Caxias do Sul: Ed. UCS, 2013.

AXELROD, L. J.; LEHMAN, D. R. Responding to environmental concerns: what factors guide individual action? Journal Environmental Psycholgy, n. 13, p. 149-159, 1993.

BENEDICTO, J. B.; SIQUEIRA, A. P. S.; MILANI, R. G. A sustentabilidade nos ambientes internos: a visão e comportamento dos estudantes. In: MOSTRA INTERNA DE TRABALHOS DE INICIAÇÃO CIENTÍFICA, 9., MOSTRA INTERNA DE INICIAÇÃO EM DESENVOLVIMENTO TECNOLÓGICO E INOVAÇÃO, 2., 2018. Maringá, PR: Unicesumar - Centro Universitário de Maringá, 2018.

BEURON, T. A. Contribuições para um modelo de universidade verde: competências e comportamentos para a sustentabilidade. 2016. 189 f. Tese (Doutorado em Administração) - Universidade Federal de Santa Maria, UFSM, 2016.

BONNES, M.; BONAIUTO, M. Environmental psychology: from spatial-physical environment to sustainable development. In: BECHTEL, R. B.; CHURCHMAN, A. (ed.). Handbook of environmental psychology. New York: Wiley, 2002. p. 28-54.

BYRNE, B. Structural equation modeling with AMOS. Basic concepts, applications and programming. Mahwah, NJ: Lawrence Erlbaum, 2001.

CALVO-SALGUEIRO, A.; AGUILAR-LUZÓN, M. DEL C.; BERRIOS-MARTOS, M. P. Comportamiento ecológico responsable: un análisis desde los valores biosféricos, sociales-altruistas y egoístas. Revista Electrónica de Investigación y Docência (Reid), n. 1, p. 11-25, 2008.

CEBDS. Conselho Empresarial Brasileiro para o Desenvolvimento Sustentável. Comunicação e Educação para a Sustentabilidade. Brasil Sustentável. 27. ed. Rio de Janeiro, 2010.

CORRAL-VERDUGO, V. Comportamento pró-ambiental: una introducción al estudio de las conductas protectoras del ambiente. Santa Cruz de Tenerife, Espanha: Resma, 2001.

CORRAL-VERDUGO, V. La definición del comportamiento proambiental. In: La Psicología Social en México, Amepso (ed.), VIII, p. 466-472, 2000.

CORRAL-VERDUGO, V.; PINHEIRO, J. Condições para o estudo do comportamento pró-ambiental. Estudos de Psicologia, n. 4, p. 7-22, 1999.

COSTA, L. S. A relação entre consumo e sustentabilidade no comportamento de jovens manauaras. 2017. 114 f. Dissertação (Mestrado) - Universidade Federal do Amazonas, Programa de Pós-Graduação em Ciências do Ambiente e Sustentabilidade na Amazônia, Manaus, AM, 2017.

DARLEY, J.; GILBERT, D. Social psychological aspects of environmental psychology. In: LINDZEY, G.; ARONSON, E.; SCHULTZ, P. W. (ed.). The handbook of social psychology. New York: Random House, 1985. v. 2. ENOKI, P. A. et al. Estratégias de marketing verde na percepção de compra dos consumidores na grande São Paulo. Revista Jovens Pesquisadores, v. 5, n. 8, p. 58, 2008.

FARIA, A. C. et al. Influência do conhecimento sobre sustentabilidade nas atitudes, comportamentos e consumo de estudantes de administração. Revista Eletrônica de Ciência Administrativa, v. 17, n. 2, p. 239260, 2018.

FEIL, A. A.; SCHREIBER, D. Sustentabilidade e desenvolvimento sustentável: desvendando as sobreposições e alcances de seus significados. Cad. EBAPE.BR, Rio de Janeiro, v. 14, n. 3, artigo 7, 2017.

FIGUEREDO, F. R.; TSARENKO, Y. Is "being green" a determinant of participation in university sustainability initiatives? International Journal of Sustainability in Higher Education, v. 14, Iss 3, p. 242-253, 2013. Disponível em: http://dx.doi.org/10.1108/IJSHE-02-2011-0017.

GARLET, V. Competências e comportamentos pró-universidade verde dos servidores em uma Instituição Federal de Ensino Superior. 2017. 98 f. Dissertação (Mestrado em Administração) - Universidade Federal de Santa Maria, UFSM, 2017.

GONÇALVES-DIAS, S. L. F. et al. Consciência ambiental: um estudo exploratório sobre suas implicações para o estudo de Administração. RAE-Eletrônica, São Paulo, v. 8, n. 1, 2009.

HAIR, J. F. Jr. et al. Análise multivariada de dados. 6. ed. Porto Alegre: Bookman, 2009.

HAIR, J. F. Jr. et al. Fundamentos de pesquisa de marketing. Porto Alegre: Bookman, 2010. 
KAISER, F. G.; FUHLER, U. Ecological behaviour's dependency on different forms of knowledge. Appl Psychol Int Rev, n. 52, p. 598-613, 2003.

KAISER, F. G.; WÖLFING, S.; FUHRER, U. Environmental attitude and ecological behaviour. Journal of Environmental Psychology, v. 19, n. 1, p. 1-19, 1999.

KARP, D. G. Values and their effect on pro-environmental behavior. Environment and Behavior, v. 28, n. 1 , p. 111-133, 1996.

LAGES, N. S.; VARGAS NETO, A. Mensurando a consciência ecológica do consumidor: um estudo realizado na cidade de Porto Alegre. In: ENCONTRO ANUAL DA ASSOCIAÇÃO BRASILEIRA DOS PROGRAMAS DE PÓS-GRADUAÇÃO EM ADMINISTRAÇÃO, 26., 2002. Salvador. Anais [...]. Salvador, 2002.

LEAL FILHO, W. Applied sustainable developement: a way forward in promoting sustainable development in higher education institutions. In: LEAL FILHO, W. (ed.). Environmental education, communication and sustainability. Frankfurt: Peter Lang, 2011.

LEHMAN, P. K.; GELLER, E. S. Behavior analysis and environmental protection: accomplishments and potential for more. Behavior and Social Issues, v. 13, p. 13-32, 2004.

MADRUGA, L. R. R. G. Comportamento coletivo e interações sociais no Comitê de Gerenciamento da Bacia Hidrográfica do Rio Santa Maria: aprendizagem social e emergência do empreendedorismo socioambiental. 2009. Tese (Doutorado) - Universidade Federal do Rio Grande do Sul, Porto Alegre, RS, 2009. Disponível em: http://www.lume.ufrgs.br/bitstream/handle/10183/15711/000680813.pdf?sequence=1. Acesso em: 23 dez. 2016.

MAGALHÃES, J. M. A responsabilidade social corporativa e seus efeitos sobre a atitude em relação à empresa e a intenção de compra dos consumidores. 2007. Dissertação (Mestrado em Administração) - Universidade do Vale do Rio Sinos, São Leopoldo, 2007.

MALONEY, M. P.; WARD, M. P.; BRAUCHT, G. N. Psychology in action: a revised scale for the measurement of ecological attitudes and knowledge. American Psychologist, Washington, DC, v. 30, n.7, p. 787-790, 1975.

MELO, D. N. B. et al. Sustentabilidade: uma investigação da atitude e do comportamento de futuros administradores. SEMEAD - SEMINÁRIOS EM ADMINISTRAÇÃO, 20., 2017, nov. 2017. ISSN 2177-3866.

MELO, D. N. B. et al. Sustentabilidade: uma investigação da atitude e do comportamento de estudantes de Administração. Contextus - Revista Contemporânea de Economia e Gestão, Especial 80 Anos FEAAC, 2018.

MOHR, L. A.; WEBB, D. J. The effects of corporate social responsibility and price on consumer responses. Journal of Consumer Affairs, v. 39, n. 1, p. 121-147, 2005.

NASCIMENTO, T. M. Examinando o domínio do comportamento pró-ambiental na promoção do bem-estar individual e coletivo. Consumer Behavior Review, v. 3, n. 1, p. 27-41. 2019a.

NASCIMENTO, T. M. Comprar bem me faz feliz? A influência do comportamento pró-ambiental no bem-estar subjetivo do consumidor. Porto Alegre: Pontifícia Universidade Católica do Rio Grande do Sul, $2019 \mathrm{~b}$. OTTMAN, J. A. Green marketing: opportunity for innovation. 2. ed. New York: BookSurge, 1998.

PASQUALI, L. Instrumentos psicológicos: manual prático de elaboração. Brasília: LabPAM, 1999.

PATO, C. M. L. Comportamento ecológico: relações com valores pessoais e crenças ambientais. 2004. Tese (Doutorado) - Universidade de Brasília, Brasília, 2004.

PATO, C.; TAMAYO, A. A escala de comportamento ecológico: desenvolvimento e validação de um instrumento de medida. Estudos de Psicologia, n. 11, p. 289-296, 2006.

PINTO, M. R.; BATINGA, G. L. Consumo consciente no contexto do consumismo moderno: algumas reflexões. Revista Gestão.Org, v. 14, n. 1, Edição Especial, 2016. p. 30-43. ISSN 1679-1827.

PRADO, J. J.; WANDER, A. E.; COSTA FILHO, B. A. Sustentabilidade nas instituições financeiras públicas e privadas: percepção, atitude e comportamento dos colaboradores. Rev. Bras. Gest. Amb. Sustent., v. 6, n. 14, p. 957-973, 2019.

RIBEIRO, J. A.; VEIGA, R. T. Proposição de uma escala de consumo sustentável. $R$. Adm., São Paulo, v. 46, n. 1, p. 45-60, jan./fev./mar. 2011.

ROBERTS, J. A. Green consumers in the 1990s: profile and implications for advertising. Journal of Business Research, New York, NY, v. 36, n. 3, p. 217-231, July 1996.

ROSA, F. C.; LEONIDIO, U. C.; JESUS, C. S. Comportamento ecologicamente consciente: um estudo dos consumidores de Petrópolis-RJ. In: SIMPÓSIO DE EXCELÊNCIA EM GESTÃO E TECNOLOGIA, 12., Resende, RJ, 2015. 
SALDANHA, G. M. Desenvolvimento local sustentável: um comportamento à sustentabilidade. 2019. Monografia de Especialização (Especialização em Gestão Pública) - Universidade Federal de Minas Gerais. Belo Horizonte, MG, 2019.

STERN, P. C. Toward a coherent theory of environmentally significant behavior. Journal of Social Issues, v. 56, p. 407-424, 2000.

STRAUGHAN, R. D.; ROBERTS, J. A. Environmental segmentation alternatives: a look at green consumer behavior in the new millennium. Journal of Consumer Marketing, West Yorkshire, UK, v. 16, n. 6, p. 558575, June 1999.

TABACHNICK, B. G.; FIDELL, L. S. Using Multivariate Statistics. 4. ed. Boston: Allyn and Bacon, 2001.

THAPA, B. Environmentalism: the relation of environmental attitudes and environmentally responsible behaviors among undergraduate students. Bulletin of Science, Technology \& Society, Thousand Oaks, CA, v. 19, n. 5, p. 426-438, Oct. 1999.

THOMASHOW, M. The nine elements of sustainable campus. USA: Massachusetts Institute of Technology, 2014.

TÓDERO, M. Consumo consciente e percepção do consumidor sobre ações corporativas vinculadas ao conceito de responsabilidade social: um estudo no setor da saúde. 2009. Dissertação (Mestrado) - Universidade de Caxias do Sul, Caxias do Sul, RS, 2009.

TRIVEDI, B. G. Food Waste Prevention and Management in Higher Education. In: LEAL FILHO, W. et al. Implementing campus greening initiatives: approaches, methods and perspectives. London: Springer, 2015. (World Sustainability Series).

UNESCO. United Nations Educational, Scientific and Cultural Organization. Shaping the future we want: With the support of UN Decade of Education for Sustainable Development (2005-2014); final report. Paris, France, 2014.

UNESCO. United Nations Educational, Scientific and Cultural Organization. United Nations Decade of Education for Sustainable Development 2005-2014: Draft International Implementation Scheme, 2005. Disponível em: http://unesdoc.unesco.org/images/0013/001399/139937e.pdf. Acesso em: 28 maio 2017.

VICENTE-MOLINA, M. A. et al. Environmental knowledge and other variables affecting pro-environmental behavior: comparison of university students from emerging and advanced countries. Journal of Cleaner Production, n. 61, p. 130-138, 2013. 